\title{
PARÂMETROS LIMNOLÓGICOS EM VIVEIROS DE PISCICULTURA SEMI-INTENSIVA DE TAMBAQUI COM ABASTECIMENTO EM DISPOSIÇÃO SEQUENCIAL
}

\author{
Fernanda Bay Hurtado* \\ Fabiano Moreira Figueiredo** \\ Rafaela Lemes da Costa*** \\ Satia Costa Bomfim***** \\ Cláudio Brandão de Queiroz $z^{* * * * *}$ \\ Wesley Paulo Pontes ${ }^{* * * * * * *}$
}

RESUMO: A piscicultura é um dos segmentos da produção animal que mais cresce no cenário mundial; neste ramo a qualidade da água é preocupação constante, pois quando esta apresenta má qualidade causa problemas na produção e pouca lucratividade. Objetivou-se avaliar a qualidade da água com base em algumas variáveis limnológicas em viveiros de piscicultura de engorda de tambaqui (Colossoma macropomum) com abastecimento em disposição sequencial. Os resultados indicaram que o regime de chuvas e o manejo durante o ciclo de produção influenciaram os parâmetros avaliados. No universo temporal da pesquisa (ago./2013 a jul./2014), os valores de $\mathrm{pH}$, amônia e nitrito estiveram de acordo com a resolução Conama 357/2005; a alcalinidade e temperatura estiveram dentro dos padrões indicados para piscicultura, o contrário para a maioria dos valores amostrados para o oxigênio dissolvido e fósforo total. Em ago./2013, mês dos menores índices pluviométricos na região, os viveiros apresentaram condutividade elétrica acima do estabelecido pela resolução Conama 357/2005; em out./2013 houve aumento expressivo da densidade da comunidade fitoplanctônica com florações pontuais, acarretando a menor transparência e oxigênio dissolvido em todos os viveiros. Os resultados indicam a necessidade de adoção sistemática de boas práticas de manejo a fim de melhorar a qualidade ambiental das criações, como também contribuir para a sustentabilidade da atividade aquícola.

PALAVRAS-CHAVE: Qualidade da água; Colossoma macropomum; Produção piscícola.

Doutora em Biologia Experimental. Docente na Universidade Federal de Rondônia - UNIR, Brasil.

E-mail: fernandabay@unir.br

** Discente de Engenharia de Pesca da Universidade Federal de Rondônia - UNIR. Bolsista de Iniciação Científica

*** Mestranda em Ciências Ambientais na Universidade Federal de Rondônia- UNIR, Brasil.

${ }^{* * * *}$ Discente do Curso de Engenharia de Pesca da Universidade Federal de Rondônia, Brasil..

${ }^{* * * * *}$ Biólogo especialista em piscicultura. Agropesca Comércio e Consultoria Ltda; Nutrizon Alimentos Ltda.

${ }^{* * * * * *}$ Discente do Curso de Engenharia de Pesca da Universidade Federal de Rondônia, Brasil. 


\title{
LIMNOLOGICAL PARAMETERS IN FISH PONDS OF TAMBAQUI SEMI-INTENSIVE CULTIVATION WITH SEQUENTIAL SUPPLY
}

\begin{abstract}
Fish culture is perhaps one of segments in animal production with high increasing rates worldwide. However, water quality is a concern due to problems in production and low profits when it is below standard. Current study focuses on water quality based on limnological variables in fish ponds for the rearing of Colossoma macropomum (tambaqui) in sequential supply. Results show that rainfall regimes and management during the production cycle affected the evaluated parameters. Research occurred between August 2013 and July 2014. Further, pH, ammonia and nitrate rates complied with Resolution by CONAMA 357/2005; alkalinity and temperature complied with fish farming standards, contrary to most dissolved oxygen and total phosphorus rates. Electrical conductivity in fish ponds in August 2013 , the month with the lowest rainfall indexes in the region, was higher than rates established by CONAMA 357/2005. The density of the phytoplankton community significantly increased in October 2013, with sparse blooming, but featuring low transparency and dissolved oxygen in all fish ponds. Results show the need for systematic introduction of Good Management Practices to improve environmental quality and contribute towards the sustainability of fish farm culture.
\end{abstract}

KEY WORDS: Water quality; Colossoma macropomum; Fish production.

\section{INTRODUÇÃO}

A produção mundial de pescado continua crescendo a um ritmo mais rápido que a população mundial, e a aquicultura continua sendo um dos setores de produção de alimentos de maior crescimento. Em 2012, a aquicultura estabeleceu alto recorde de produção e atualmente fornece quase metade dos peixes utilizados para o consumo humano, com aumento estimado em 62\% para 2030 pela estabilização do desempenho da pesca, e a aquicultura pode gerar benefícios duradouros à segurança alimentar mundial e ao crescimento econômico se for desenvolvida e praticada de maneira responsável (FAO, 2014).

O Brasil possui grande potencial para o desenvolvimento da aquicultura

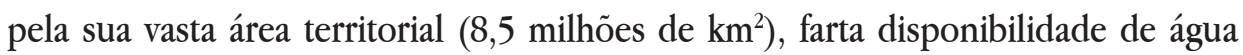
potável, extensa orla marítima $(8.698 \mathrm{~km})$ e condições climáticas favoráveis. Nesse 
sentido, ainda há grande potencial para a aquicultura ser explorada, gerando novos empregos, renda e alimentos de alto valor biológico (IBGE, 2013). Com relação ao ano de 2014 a produção total da piscicultura aumentou em $20,9 \%$, o que correspondeu a 474,33 mil toneladas, e a região Norte passou para a liderança entre as demais regióes; essa produtividade foi impulsionada pelo Estado de Rondônia, que ascendeu para a primeira posição do ranking com a despesca de 75,02 mil toneladas de peixes, o que correspondeu a $15,8 \%$ da produção entre as Unidades da Federação (IBGE, 2014).

A implantação de uma piscicultura deve ser bem planejada, onde devem ser elencadas as características e condições da propriedade para a identificação das vantagens e restrições para a sua execução. Para tal devem-se considerar os aspectos da água e do solo, a incidência da radiação solar, o local escolhido para a instalação da atividade na propriedade, o qual deve possuir declividade adequada e não sofrer a influência dos rios durante as cheias, possuir fácil acesso, além de favorecer a construção de barragem e tanques, a água deve ter boa qualidade, deve ser poluída e ser renovada periódicamente; outro fator importante é a fonte de abastecimento, a qual preferencialmente deve estar dentro dos limites da propriedade, tais como a nascente do rio, riacho ou igarapé a ser utilizado (BARBOSA; SOUSA NETO, 2014)

Dentre os fatores que levam à degradação da qualidade da água no sistema de produção piscícola, os quais modificam a ecologia dos sistemas de cultivo, destaca-se a densidade e a espécie de peixe utilizadas, assim como o manejo aplicado durante o ciclo de produção (adubação e arraçoamento). Esses fatores influenciam, principalmente, no oxigênio dissolvido, nitrogênio amoniacal, teor de fosfato, condutividade elétrica, matéria orgânica, potencial hidrogeniônico, biomassa bentônica e planctônica (MINUCCI; PINESE; ESPÍNDOLA, 2005).

Com a evolução da questão ambiental e das condições que o planeta apresenta a criação racional de organismos aquáticos, a aquicultura é uma atividade economicamente emergente na competição pelo recurso água (ELER; MILANI, 2007). Atualmente, a aquicultura enfrenta o desafio de moldar-se ao conceito de sustentabilidade, o que implica em agregar novos valores à produção de conhecimento e às boas práticas do setor (ELER; MILANI, 2007).

A produção de peixes pela piscicultura tem sido uma alternativa ambientalmente adequada para preservação de recursos naturais quando comparada 
à pesca extrativista, e também ser uma alternativa de fonte de renda, especialmente, para populações que dependem da pesca em diversos níveis, além dos pequenos produtores rurais. Entretanto, para que esta atividade seja bem sucedida é necessário o conhecimento técnico em diversas áreas, dentre estas a limnologia e, especificamente, a qualidade da água (PEREIRA et al., 2016).

$\mathrm{O}$ objetivo da presente pesquisa foi avaliar alguns parâmetros limnológicos abióticos de viveiros de piscicultura de engorda de tambaqui (Colossoma macropomum) com abastecimento sequencial.

\section{MATERIAL E MÉTODOS}

\section{1 ÁREA DE ESTUDO}

A pesquisa foi realizada na Piscicultura Santa Helena, localizada no $\mathrm{km} 14$, TN 13, gleba 4, no distrito de Tancredópolis, município de Alvorada d'Oeste, Rondônia, Brasil (Figura 1). A área da propriedade destinada para atividade de piscicultura é de 3,0 hectares de lâmina d'água, que conta com sete viveiros para engorda de tambaqui e um viveiro berçário para alevinos, os quais são mantidos por um período de 45 dias e, posteriormente, são transferidos para os viveiros de engorda. Todos os viveiros apresentam fundo natural e profundidade média que varia entre 1,50 - 1,80 m e são abastecidos com água procedente de nascentes da propriedade. 


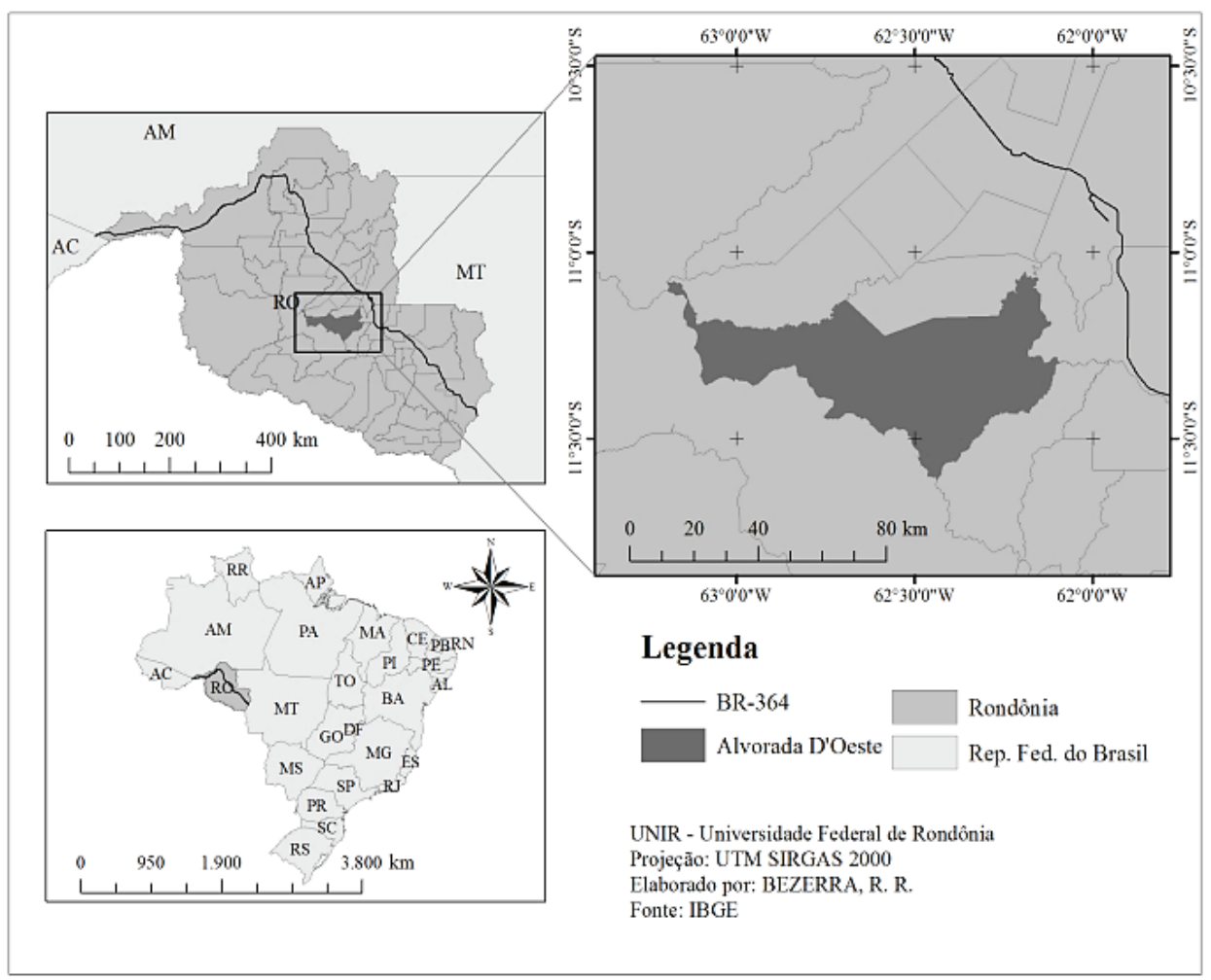

Figura 1. Localização da Piscicultura Santa Helena

\subsection{MÉTODO DE COLETA}

As amostragens foram realizadas bimensalmente entre o período de agosto de 2013 a maio de 2014, compreendendo as estações seca (ago. e out./2013 e jul./2014) e chuvosa (dez., fev. e maio/2014) (FERNANDES; GUIMARÃES, 2003). As amostragens ocorreram na represa de abastecimento (cuja água é destinada ao abastecimento dos viveiros) e nos viveiros de engorda, a $36 \mathrm{~cm}$ de profundidade, de forma alternada viveiro 1 (T-1), viveiro 3 (T-3) e viveiro 5 (T-5) (Figura 2), sendo um ponto na proximidade da tubulação de abastecimento e o outro na tubulação de escoamento.

Para a seleção dos pontos amostrais levou-se em consideração que a entrada de água nos viveiros de engorda ocorre em disposição sequencial, em que a represa 
de abastecimento fornece a água do primeiro viveiro e a partir daí essa água vai abastecendo os demais.

Todas as amostras que necessitaram ser transportadas para posterior análise foram acondicionadas em frascos de vidro borosilicato âmbar, devidamente identificadas e refrigeradas para preservação, conforme Guia Nacional de Coleta e Preservação de Amostras (Cetesb, 2011).

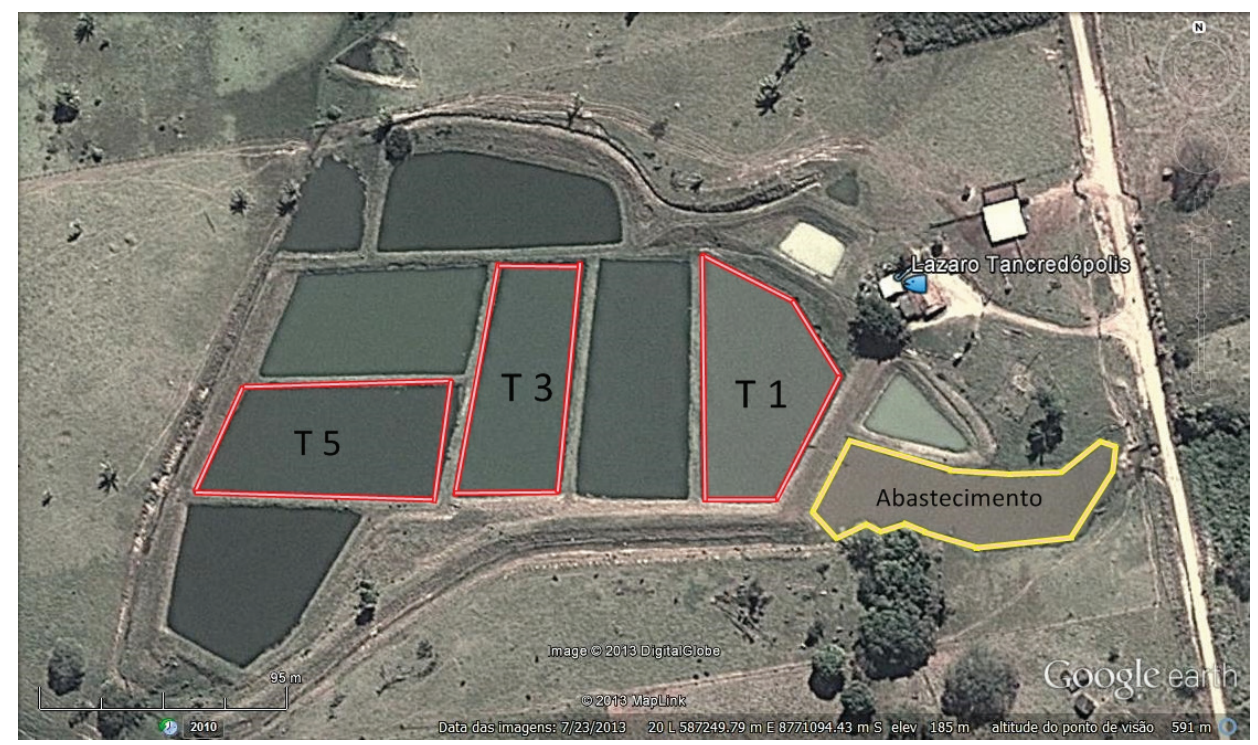

Figura 2. Locais de amostragem na Piscicultura Santa Helena

Fonte: Adaptado do Google Earth (acesso em: 23/03/2016).

\subsection{NÁLISE DOS PARÂMETROS LIMNOLÓGICOS}

A aferição da temperatura $\left({ }^{\circ} \mathrm{C}\right)$ e do oxigênio dissolvido (mg.L $\left.{ }^{-1}\right)$ aconteceu no momento da coleta com o uso de sonda multiparâmetro (YSI pro20), o pH com pHmetro (YSI pH10A) e a transparência (cm) com disco de Secchi. Os demais parâmetros como alcalinidade $\left(\mathrm{mg} . \mathrm{L}^{-1}\right)$, condutividade $\left(\mu \mathrm{S} . \mathrm{cm}^{-1}\right)$, amônia não ionizada (mg. $\left.\mathrm{L}^{-1}\right)$ e ionizada (mg. $\left.\mathrm{L}^{-1}\right)$, nitrito (mg. $\left.\mathrm{L}^{-1}\right)$ e fósforo total (mg. $\left.\mathrm{L}^{-1}\right)$ foram realizadas no Laboratório de Análises Físico-Químicas e Microbiológicas no campus de Presidente Médici da Universidade Federal de Rondônia, com fotômetro (HI 
83099 HANNA) e kit de análise específico para cada parâmetro. Os resultados obtidos foram comparados com o estabelecido pela resolução Conama 357/2005 (CONAMA, 2005), verificando os dados obtidos conforme sua classe de enquadramento de uso (piscicultura).

Os dados foram tabulados e submetidos a testes no programa estatístico OriginPro 8, sendo o teste Shapiro-Wilk (normalidade) para aceitação das variâncias, Análise de Variância (Anova) para detectar a existência de diferenças significativas, seguida por teste de Tukey $\mathrm{p}=0,05$ para verificar em quais grupos existiram as diferenças.

\section{RESULTADOS E DISCUSSÃO}

O conhecimento dos valores dos parâmetros físico-químicos da água no sistema de cultivo é um fator crucial para o sucesso na produção de peixes além de auxiliar produtores, técnicos e pesquisadores bem como também preservar os corpos hídricos. Em 2005 foi criada a resolução Conama 357/2005 que estabelece parâmetros físico-químicos para corpos de água onde haja pesca ou cultivo de organismos para fins de consumo intensivo, pois uma das preocupações referentes ao meio ambiente aquático é a eutrofização; na produção piscícola esta é causada principalmente pelo arraçoamento excessivo, por rações de baixa qualidade ou de balanceamento inadequados de nutrientes, acarretando em carências nutricionais e consequente enriquecimento hídrico, levando ao desequilíbrio do meio causando estresse e dificuldades de desenvolvimento dos peixes, ou até morte.

Houve variação da temperatura da água (Ta), com diferença de $1,3^{\circ} \mathrm{C}$ entre a maior e menor temperatura, as médias para a represa de abastecimento e viveiros nos períodos de coleta não obtiveram diferença significativa indicando que a temperatura não variou de maneira acentuada entre pontos amostrados (Tabela 1), também não houve diferença significativa entre a média dos meses amostrados (Tabela 2).

Os valores registrados estão dentro dos padrões sugeridos por Boyd e Tucker (1992) específicos para aquicultura, e superiores a indicada por Watanabe 
et al. (2007), os quais afirmam que peixes de águas tropicais geralmente vivem bem com temperaturas entre $20-28^{\circ} \mathrm{C}$, sendo que acima de $28^{\circ} \mathrm{C}$ estes perdem totalmente $\mathrm{o}$ apetite, podendo ocorrer mortalidade em temperaturas superiores a $32^{\circ} \mathrm{C}$.

Condições semelhantes foram observadas por Craef et al. (1987) e Resende et al. (1985), criando peixes em represas na região Amazônica, em temperaturas entre $27^{\circ} \mathrm{C}$ a $31^{\circ} \mathrm{C}$, onde estes consideram propícias para a produção de organismos tropicais. Já para Faria et al. (2013), a temperatura ideal para o desenvolvimento de peixes tropicais em viveiros de engorda situa-se entre $25^{\circ} \mathrm{C}$ e $32^{\circ} \mathrm{C}$. Para peixes tropicais, valores abaixo dos encontrados podem causar problemas na alimentação, reprodução e desenvolvimento das espécies, comprometendo o sistema imunológico e deixando-os susceptíveis a doenças (SILVA, FERREIRA; LOGATO, 2001).

Grande parte dos valores de oxigênio dissolvido (OD) registrados estava abaixo do estabelecido pela resolução Conama 357/2005, a qual sugere valores superiores a 5 mg.L-1 . Faria et al. (2013) também classificam valores acima de 5 mg.L: ${ }^{1}$ de OD na água como ideais, pois segundo o autor em valores entre $1 \mathrm{a} 5 \mathrm{mg} . \mathrm{L}^{-1}$ os peixes sobrevivem, mas pode haver diminuição das taxas de crescimento caso a exposição seja muito prolongada, e quando inferior a $1 \mathrm{mg} . \mathrm{L}^{-1}$ é letal se exposto por tempo prolongado.

Tabela 1. Média e desvio-padrão dos parâmetros limnológicos da água dos viveiros amostrados por ponto de coleta

(Continua)

\begin{tabular}{|c|c|c|c|c|c|c|c|}
\hline \multirow{2}{*}{ Parâm. } & \multirow{2}{*}{$\frac{\text { Abst }}{\text { Saída }}$} & \multicolumn{2}{|c|}{ Viveiro 1} & \multicolumn{2}{|c|}{ Viveiro 3} & \multicolumn{2}{|c|}{ Viveiro 5} \\
\hline & & Entrada & Saída & Entrada & Saída & Entrada & Saída \\
\hline $\mathrm{Ta}$ & $27,8 \pm 0,7$ & $\begin{array}{c}29,1 \pm \\
0,9\end{array}$ & $29,0 \pm 0,9$ & $\begin{array}{c}28,9 \pm \\
0,7\end{array}$ & $\begin{array}{c}28,8 \pm \\
0,7\end{array}$ & $28,9 \pm 0,8$ & $29,1 \pm 0,8$ \\
\hline OD & $2,5 \pm 0,8^{\mathrm{a}}$ & $3,6 \pm 0,9$ & $3,2 \pm 1,0^{\mathrm{b}}$ & $4,0 \pm 2,2$ & $\begin{array}{c}3,9 \pm \\
2,0\end{array}$ & $4,5 \pm 1,7^{\mathrm{a}, \mathrm{b}}$ & $5,2 \pm 1,5^{\mathrm{a}, \mathrm{b}}$ \\
\hline $\mathrm{pH}$ & $6,9 \pm 0,3^{\mathrm{a}}$ & $7,3 \pm 0,7^{\mathrm{a}}$ & $7,2 \pm 0,6^{b}$ & $7,5 \pm 0,4$ & $\begin{array}{c}7,7 \pm \\
0,3\end{array}$ & $7,9 \pm 0,8^{a, b}$ & $7,8 \pm 0,8^{a, b}$ \\
\hline Trp. & $\begin{array}{c}30,8 \pm \\
10,1^{a, b, c, d}\end{array}$ & $\begin{array}{l}28,6 \pm \\
3,3^{a, b, c, d}\end{array}$ & $\begin{array}{l}29,5 \pm \\
4,1^{a, b, c, d}\end{array}$ & $\begin{array}{c}23,8 \pm \\
7,5^{\mathrm{a}}\end{array}$ & $\begin{array}{c}23,6 \pm \\
7,3^{\mathrm{b}}\end{array}$ & $24,0 \pm 7,5^{c}$ & $23,0 \pm 6,2^{\mathrm{d}}$ \\
\hline
\end{tabular}


(Conclusão)

\begin{tabular}{lccccccc}
\hline \multirow{2}{*}{ Parâm.m. } & Abst & \multicolumn{2}{c}{ Viveiro 1} & \multicolumn{2}{c}{ Viveiro 3 } & \multicolumn{2}{c}{ Viveiro 5 } \\
\cline { 2 - 7 } & Saída & Entrada & Saída & Entrada & Saída & Entrada & Saída \\
\hline \multirow{2}{*}{ Alc. } & $77,5 \pm$ & $80,4 \pm$ & $88,9 \pm$ & $100,7 \pm$ & $107,5 \pm$ & $67,5 \pm$ & $96,3 \pm$ \\
& $24,0^{\mathrm{a}}$ & $27^{\mathrm{b}}$ & $25,7^{\mathrm{a}, \mathrm{b}}$ & $45,3^{\mathrm{a}, \mathrm{b}, \mathrm{c}}$ & $53,1^{\mathrm{a}, \mathrm{b}, \mathrm{c}, \mathrm{d}}$ & $18,6^{\mathrm{b}, \mathrm{c}}$ & $20,6^{\mathrm{a}, \mathrm{c}, \mathrm{d}}$ \\
& & & & & & & \\
$\mathrm{CE}$ & $88,3 \pm$ & $99,3 \pm$ & $98,5 \pm$ & $103,6 \pm$ & 105,9 & $118,7 \pm$ & $120,2 \pm$ \\
& $15,6^{\mathrm{a}}$ & $12,5^{\mathrm{a}, \mathrm{b}}$ & $10,6^{\mathrm{c}}$ & $16,2^{\mathrm{a}, \mathrm{d}}$ & $\pm 15,4^{\mathrm{a}, \mathrm{e}}$ & $19,9^{\mathrm{a}, \mathrm{b}, \mathrm{c}, \mathrm{d}, \mathrm{e}}$ & $18,3^{\mathrm{a}, \mathrm{b}, \mathrm{c}, \mathrm{d}, \mathrm{e}}$ \\
& $0,55 \pm$ & $1,05 \pm$ & $0,83 \pm$ & $0,66 \pm$ & $0,65 \pm$ & $0,70 \pm$ & \\
$\mathrm{NH}_{3}$ & $0,5^{\mathrm{a}}$ & $0,6^{\mathrm{a}, \mathrm{b}, \mathrm{c}, \mathrm{d}, \mathrm{e}}$ & $0,7^{\mathrm{a}, \mathrm{b}, \mathrm{c}, \mathrm{d}}$ & $0,5^{\mathrm{a}, \mathrm{b}}$ & $0,5^{\mathrm{c}}$ & $0,6^{\mathrm{a}, \mathrm{d}}$ & $0,73 \pm 0,5^{\mathrm{a}, \mathrm{e}}$ \\
& $0,59 \pm$ & $1,11 \pm$ & $0,88 \pm$ & $0,70 \pm$ & $0,69 \pm$ & $0,74 \pm$ & \\
$\mathrm{NH}_{4}{ }^{1+}$ & $0,5^{\mathrm{a}}$ & $0,5^{\mathrm{a}, \mathrm{b}, \mathrm{c}, \mathrm{d}, \mathrm{e}}$ & $0,6^{\mathrm{a}, \mathrm{b}, \mathrm{c}, \mathrm{d}}$ & $0,5^{\mathrm{a}, \mathrm{b}}$ & $0,5^{\mathrm{a}, \mathrm{c}}$ & $0,6^{\mathrm{a}, \mathrm{d}}$ & $0,83 \pm 0,7^{\mathrm{a}, \mathrm{e}}$ \\
& $0,02 \pm$ & $0,03 \pm$ & $0,03 \pm$ & $0,01 \pm$ & $0,01 \pm$ & $0,01 \pm$ & \\
$\mathrm{NO}_{2}{ }^{1 .}$ & 0,01 & $0,02^{\mathrm{a}}$ & $0,02^{\mathrm{b}}$ & $0,08^{\mathrm{a}, \mathrm{b}}$ & $0,0^{\mathrm{a}, \mathrm{b}}$ & $0,0^{\mathrm{a}, \mathrm{b}}$ & $0,01 \pm 0,0^{\mathrm{a}, \mathrm{b}}$ \\
& $0,42 \pm$ & $0,35 \pm$ & $0,08 \pm$ & $0,91 \pm$ & $0,32 \pm$ & $0,13 \pm$ & $0,36 \pm$ \\
$\mathrm{P}_{\mathrm{t}}$ & $0,3^{\mathrm{a}, \mathrm{b}, \mathrm{c}, \mathrm{d}}$ & $0,2^{\mathrm{b}, \mathrm{c}, \mathrm{d}}$ & $0,04^{\mathrm{a}, \mathrm{b}, \mathrm{c}}$ & $0,6^{\mathrm{a}, \mathrm{b}, \mathrm{c}, \mathrm{d}}$ & $0,2^{\mathrm{a}, \mathrm{c}, \mathrm{d}, \mathrm{e}}$ & $0,08^{\mathrm{a}, \mathrm{b}, \mathrm{d}, \mathrm{d}, \mathrm{f}}$ & $0,24^{\mathrm{c}, \mathrm{d}, \mathrm{f}}$ \\
\hline
\end{tabular}

Onde: Parâm. $=$ Parâmetros analisados, Abast. $=$ Represa de abastecimento, Ta $=$ Temperatura da água $\left({ }^{\circ} \mathrm{C}\right) ; \mathrm{OD}=$ Oxigênio dissolvido $\left(\mathrm{mg} . \mathrm{L}^{-1}\right) ; \mathrm{pH}=$ Potencial hidrogeniônico, Trp. = Transparência $(\mathrm{cm})$, Alc $=$ Alcalinidade $\left(\mathrm{mg} \cdot \mathrm{L}^{-1}\right.$ de $\left.\mathrm{CaCO}_{3}\right), \mathrm{CE}=$ Condutividade elétrica $\left(\mu \mathrm{S} . \mathrm{cm}^{-1}\right), \mathrm{NH}_{3}=$ Amônia não ionizada $\left(\mathrm{mg} . \mathrm{L}^{-1}\right) ; \mathrm{NH}_{4}{ }^{1+}=$ Amônia ionizada $\left(\mathrm{mg} \cdot \mathrm{L}^{-1}\right) ; \mathrm{NO}_{3}{ }^{1-}=$ nitrito $\left(\mathrm{mg} \cdot \mathrm{L}^{-1}\right) ; \mathrm{P}_{\mathrm{t}}=$ Fósforo total (mg. $\left.\mathrm{L}^{-1}\right)$. As médias seguidas pela mesma letra na linha diferem significativamente em nível de 5\%, pelo Teste de Tukey.

Os resultados obtidos no parâmetro $\mathrm{pH}$ variaram de pouco ácido a alcalino $(6,4$ a 9,1). O valor mínimo foi registrado em maio/2014, no viveiro 1 ( $\mathrm{pH}=6,4)$, e o valor máximo no viveiro 5, em fev./2014 ( $\mathrm{pH}=9,1)$, havendo diferença significativa entre as médias das amostras da represa de abastecimento e a entrada do viveiro 3 a entrada do viveiro 5 e as médias do viveiro 1 e do mês de dez./2013 e jan./2014, de forma que a elevação do pH no mês de fev./2014 está correlacionada com a calagem e adubação dos viveiros ocorrida no final de jan./2014 para o início do novo ciclo de produção (Tabela 2). 
Tabela 2. Média e desvio-padrão dos parâmetros físico-químicos por mês dos viveiros amostrados

\begin{tabular}{|c|c|c|c|c|c|c|}
\hline Parâmetros & Ago./2013 & Out./2013 & Dez./2013 & Fev./2014 & Maio/2014 & Jul./2014 \\
\hline $\mathrm{Ta}$ & $28,6 \pm 0,3$ & $28,7 \pm 0,6$ & $29,6 \pm 0,8$ & $27,6 \pm 0,4$ & $29,1 \pm 0,6$ & $27,2 \pm 0,9$ \\
\hline OD & $\begin{array}{l}3,7 \pm \\
1,5^{\mathrm{a}, \mathrm{b}}\end{array}$ & $2,1 \pm 1,7^{a}$ & $1,7 \pm 0,5^{\mathrm{a}, \mathrm{b}, \mathrm{c}}$ & $2,1 \pm 1,1^{\mathrm{a}}$ & $3,0 \pm 0,7^{\mathrm{b}}$ & $3,2 \pm 1,2^{\mathrm{b}, \mathrm{c}}$ \\
\hline $\mathrm{pH}$ & $7,4 \pm 0,3$ & $7,4 \pm 0,6$ & $7,4 \pm 0,2$ & $8,2 \pm 0,7$ & $7,6 \pm 0,3$ & $7,3 \pm 0,6$ \\
\hline Trp. & $\begin{array}{l}27,1 \pm \\
5,6^{\mathrm{a}}\end{array}$ & $\begin{array}{l}17,6 \pm \\
5,9^{a, b}\end{array}$ & $23,8 \pm 4,7$ & $30,1 \pm 4,5^{b}$ & $32,1 \pm 3,8^{b}$ & $25,2 \pm 7,1^{b}$ \\
\hline Alc. & $\begin{array}{l}88,6 \pm \\
19,2^{\mathrm{a}}\end{array}$ & $\begin{array}{l}75,4 \pm \\
17,4^{\mathrm{a}, \mathrm{b}}\end{array}$ & $78,2 \pm 5,7^{\mathrm{c}}$ & $\begin{array}{l}127,9 \pm \\
8,8^{\text {a,b,c,d,e }}\end{array}$ & $\begin{array}{l}113,7 \pm \\
10,2^{\mathrm{a}, \mathrm{b}, \mathrm{c}, \mathrm{d}}\end{array}$ & $\begin{array}{l}98,6 \pm \\
11,3^{b, \mathrm{~b}, \mathrm{~d}, \mathrm{e}}\end{array}$ \\
\hline $\mathrm{CE}$ & $\begin{array}{l}102,5 \pm \\
9,2^{\mathrm{a}}\end{array}$ & $\begin{array}{l}106,4 \pm \\
8,7^{b}\end{array}$ & $\begin{array}{l}111,4 \pm \\
14,4^{\mathrm{a}}, \mathrm{c}\end{array}$ & $\begin{array}{r}129,124 \pm \\
42,8^{\mathrm{a}, \mathrm{b}, \mathrm{c}, \mathrm{d}}\end{array}$ & $\begin{array}{l}115,2 \pm \\
26,4^{\mathrm{a}, \mathrm{b}}\end{array}$ & $\begin{array}{l}108,3 \pm \\
10,5^{\mathrm{d}}\end{array}$ \\
\hline $\mathrm{NH}_{3}$ & $\begin{array}{l}0,99 \pm \\
0,3^{\mathrm{a}}\end{array}$ & $0,6 \pm 0,7^{\mathrm{b}, \mathrm{d}}$ & $\begin{array}{l}1,11 \pm \\
0,2^{\mathrm{b}, \mathrm{c}}\end{array}$ & $\begin{array}{l}0,38 \pm \\
0,1^{1, b, c, d, d}\end{array}$ & $0,77 \pm 0,2^{\mathrm{c}, \mathrm{d}}$ & $0,85 \pm 0,3^{\mathrm{d}}$ \\
\hline $\mathrm{NH}_{4}{ }^{1+}$ & $\begin{array}{l}1,04 \pm \\
0,3^{3, d}\end{array}$ & $1,13 \pm 0,8^{b}$ & $\begin{array}{l}1,28 \pm \\
0,3^{\mathrm{a}, \mathrm{b}, \mathrm{c}}\end{array}$ & $\begin{array}{l}0,49 \pm \\
0,2^{2, b, c, d, d}\end{array}$ & $\begin{array}{l}0,65 \pm \\
0,3^{3}, \mathrm{~b}, \mathrm{c}, \mathrm{d}\end{array}$ & $0,98 \pm 0,4^{\mathrm{d}}$ \\
\hline $\mathrm{NO}_{2}{ }^{1 \cdot}$ & $\begin{array}{l}0,01 \pm \\
0,0^{\mathrm{a}}\end{array}$ & $\begin{array}{l}0,03 \pm \\
0,02^{\mathrm{b}}\end{array}$ & $\begin{array}{l}0,06 \pm \\
0,0^{\mathrm{a}, \mathrm{b}, \mathrm{c}, \mathrm{d}, \mathrm{e}}\end{array}$ & $0,03 \pm 0,0^{c}$ & $0,01 \pm 0,0^{\mathrm{d}}$ & $0,01 \pm 0,0^{\mathrm{e}}$ \\
\hline $\mathrm{Pt}$ & $\begin{array}{l}0,34 \pm \\
0,07^{\mathrm{a}}\end{array}$ & $\begin{array}{l}0,46 \pm \\
0,05^{\mathrm{b}}\end{array}$ & $\begin{array}{l}0,62 \pm \\
0,07^{\mathrm{a}, \mathrm{b}, \mathrm{c}}\end{array}$ & $\begin{array}{l}0,11 \pm \\
0,04^{\mathrm{a}, \mathrm{b}, \mathrm{c}, \mathrm{d}}\end{array}$ & $\begin{array}{l}0,19 \pm \\
0,08^{\mathrm{a}, \mathrm{b}, \mathrm{c}}\end{array}$ & $\begin{array}{l}0,26 \pm \\
0,04^{\mathrm{b}, c, \mathrm{~d}}\end{array}$ \\
\hline
\end{tabular}

Onde: Abast. $=$ Represa de abastecimento, $\mathrm{Ta}=$ Temperatura da água $\left({ }^{\circ} \mathrm{C}\right) ; \mathrm{OD}=$ Oxigênio dissolvido $\left(\mathrm{mg} . \mathrm{L}^{-1}\right) ; \mathrm{pH}=$ Potencial hidrogeniônico, Trp. $=$ Transparência $(\mathrm{cm})$, Alc $=$ Alcalinidade $\left(\mathrm{mg} . \mathrm{L}^{-1} \mathrm{de} \mathrm{CaCO}_{3}\right), \mathrm{CE}=$ Condutividade elétrica $\left(\mu \mathrm{S} . \mathrm{cm}^{-1}\right), \mathrm{NH}_{3}=$ Amônia não ionizada $\left(\mathrm{mg} . \mathrm{L}^{-1}\right)$; $\mathrm{NH}_{4}^{1+}=$ Amônia ionizada $\left(\mathrm{mg} \cdot \mathrm{L}^{-1}\right) ; \mathrm{NO}_{3}{ }^{1 \cdot}=$ nitrito $\left(\mathrm{mg} \cdot \mathrm{L}^{-1}\right) ; \mathrm{P}_{\mathrm{t}}=$ Fósforo total $\left(\mathrm{mg} \cdot \mathrm{L}^{-1}\right)$. As médias seguidas pela mesma letra na linha diferem significativamente em nível de 5\%, pelo Teste de Tukey.

Os valores de pH estão de acordo com os valores de referência (entre 6,0 e 9,0) propostos pela resolução Conama 357/2005 para águas doces classe 2, e próximos aos padrões específicos para aquicultura, citados por Boyd (2003) que são de $\mathrm{pH}$ entre 7,0 e 9,0 para a piscicultura.

Segundo Ceccarelli, Senhorini e Volpato (2000), o pH ótimo para a produção de peixes tropicais deve permanecer entre 7,0 e 8,0, mas Resende et al. (1985) também obtiveram bons resultados produzindo peixes com $\mathrm{pH}$ variando de 4,9 a 8,3. Aride, Roubach e Val (2007) concluíram que a baixa sensibilidade do 
tambaqui a valores ácidos de $\mathrm{pH}$ pode ser considerada como uma evolução da espécie pela acidez das águas na região Amazônica. Baldisseroto (2013a) indica como faixa de pH recomendada para a produção de peixes de 6,5 a 9,0, sendo estes valores considerados apropriados para a criação de espécies comerciais de água doce, conforme foi observado nos resultados desta pesquisa.

A transparência variou entre 13 a $40 \mathrm{~cm}$, o menor valor foi registrado em out./2013, no viveiro $3(13 \mathrm{~cm})$, sendo os menores valores para os demais viveiros também registrados neste mês, os quais se atribui ao bloom de algas observado no dia da coleta, o maior valor para este parâmetro foi obtido na represa de abastecimento no mês de maio/2014 $(40 \mathrm{~cm})$.

A diminuição da transparência de um viveiro para o outro pode ser atribuída ao sistema de abastecimento tipo cascata (Tabela 1); também houve correlação inversa entre a transparência e a condutividade elétrica (Figura 2), o que é atribuído ao tipo de sistema de abastecimento desta piscicultura, de modo que a transparência no decorrer dos viveiros diminui enquanto a condutividade elétrica aumenta, indicando assim grande quantidade de matéria orgânica em decomposição.

Segundo Faria et al. (2013), os valores ideais para este parâmetro na piscicultura são entre 30 e $60 \mathrm{~cm}$, o que indica a existência de quantidade adequada de plâncton (água levemente esverdeada), pois a baixa transparência $(<30 \mathrm{~cm})$ pode indicar excesso de plâncton, material em suspensão ou revolvimento do fundo, o que impede a penetração da luz, diminuindo a produção de oxigênio realizada pelas microalgas (fitoplâncton). Entretanto, a alta transparência $(>60 \mathrm{~cm})$ indica falta de plâncton, que pode ocasionar grande variação de $\mathrm{pH}$ ao longo do dia, trazendo consequências prejudiciais à piscicultura, além de favorecer o aparecimento de algas filamentosas e plantas aquáticas que dificultam o manejo no momento da despesca.

A condutividade elétrica (CE) variou entre 66,0 a $155,3 \mu \mathrm{S} . \mathrm{cm}^{-1}$, o menor valor $\left(66,0 \mu \mathrm{S} . \mathrm{cm}^{-1}\right)$ ocorreu em out./2013 no abastecimento e o máximo $(155,2$ $\mu \mathrm{S} . \mathrm{cm}^{-1}$ ) em ago./2013 na saída o viveiro 5 (Tabela 2), havendo diferença significativa entre as médias da entrada do viveiro 3 e represa de abastecimento e saída do viveiro 5 (Tabela 1).

Segundo a resolução Conama 357/2005, a CE não deve ultrapassar 100 $\mu \mathrm{S} . \mathrm{cm}^{-1}$; nesta pesquisa a maioria dos valores foi superior ao estabelecido, o que 
indica relação inversamente proporcional aos níveis de pluviosidade (Figura 7), entretanto, a elevação dos seus níveis entre os tanques e ao decorrer deste estudo pode estar associada ao aumento da matéria orgânica na água, proveniente das excretas dos peixes e resto de ração não consumida, contribuindo para o acúmulo de íons no ambiente de cultivo (ITUASSU et al., 2004); tempo de residência da água, aumento dos teores de dureza e a realização de calagem (OLIVEIRA et al., 2010).

A alcalinidade variou entre 60 a 187,5 mg. $\mathrm{L}^{-1}$ de $\mathrm{CaCO}_{3}$, estando dentro dos valores indicados por Queiroz e Boeira (2006), já Boyd (1992) indica que para a piscicultura são recomendados valores de alcalinidade da água entre 25 e 100 mg. $\mathrm{L}^{-1}$, o valor mínimo de alcalinidade ocorreu em dez./2013, na represa de abastecimento e o valor máximo ocorreu em fev./2014 na saída do viveiro 3 (Tabela 1), havendo significância apenas entre as médias dos viveiros no mês de ago./2013 e fev./2014 (tabela 2).

Segundo Queiroz e Boeira (2006), uma das formas de minimizar variações bruscas diárias de $\mathrm{pH}$ em tanques de piscicultura é manter a alcalinidade da água em valores acima de 40 até $250 \mathrm{mg} . \mathrm{L}^{-1}$ de $\mathrm{CaCO}_{3}$; este efeito é denominado de tampão. $\mathrm{Na}$ piscicultura pode-se elevar o $\mathrm{pH}$ pela aplicação de calcário agrícola neutralizando a acidez da água. Este efeito tampão pode ser observado nas amostras de fev./2014 (Figura 3) pela utilização da calagem em jan./2014 para o início do novo ciclo de produção. 


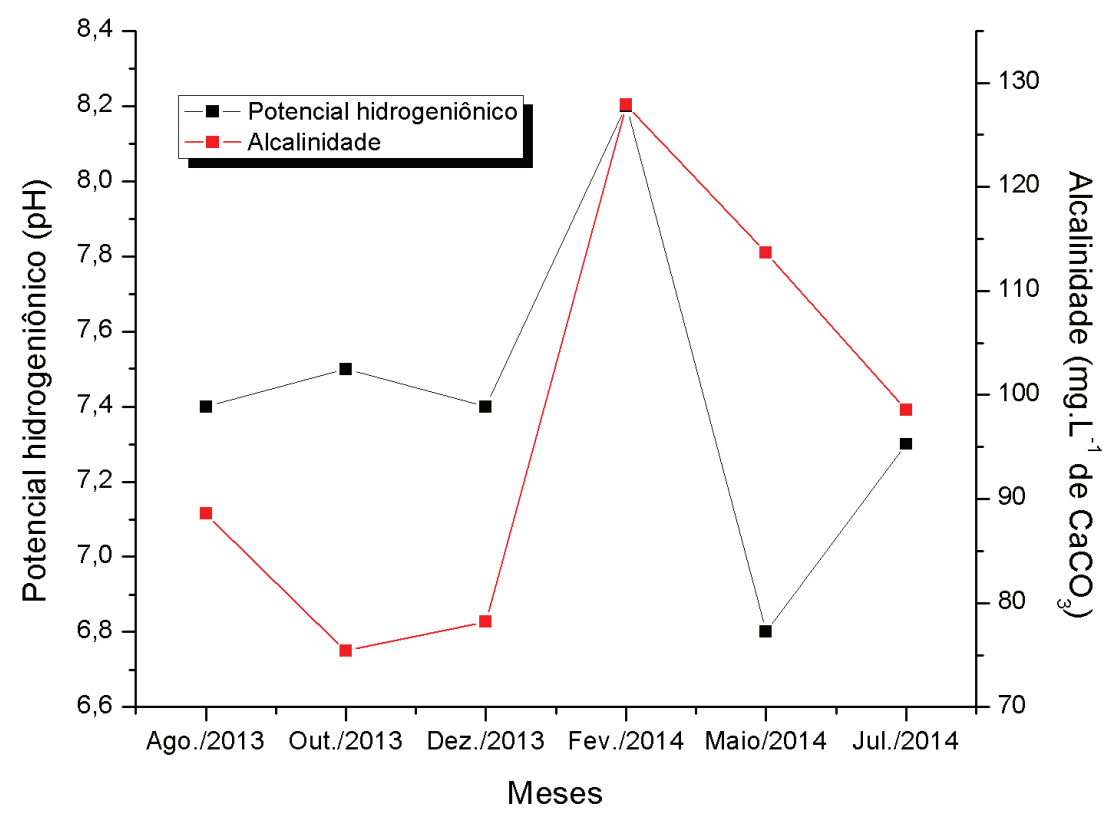

Figura 3. Correlação entre os valores de potencial hidrogeniônico e alcalinidade

O valor médio da CE por viveiro indica que a concentração iônica aumentou gradativamente da represa de abastecimento até viveiro 5 (Tabela 1), o que é atribuído a forma do sistema de abastecimento dos viveiros não individualizado, ou seja, quando a água de um viveiro abastece o outro, os íons advindos dos viveiros a montante vão se acumulando no viveiro a jusante, atribuindo sempre ao viveiro seguinte água de baixa qualidade para a produção.

Correlaciona-se os valores da CE com as cargas de íons bicarbonatos $\left(\mathrm{HCO}_{3}{ }^{1-}\right)$, pois após a calagem dos viveiros para início do novo ciclo de produção os valores aumentaram significativamente. Ainda, segundo Tundisi e Straskraba (1995), este parâmetro relaciona-se com a presença de íons dissolvidos no ambiente como partículas carregadas eletricamente, e quanto maior for a quantidade de íons dissolvidos na água maior será sua condutividade elétrica (Tabela 1 e Figura 4). 


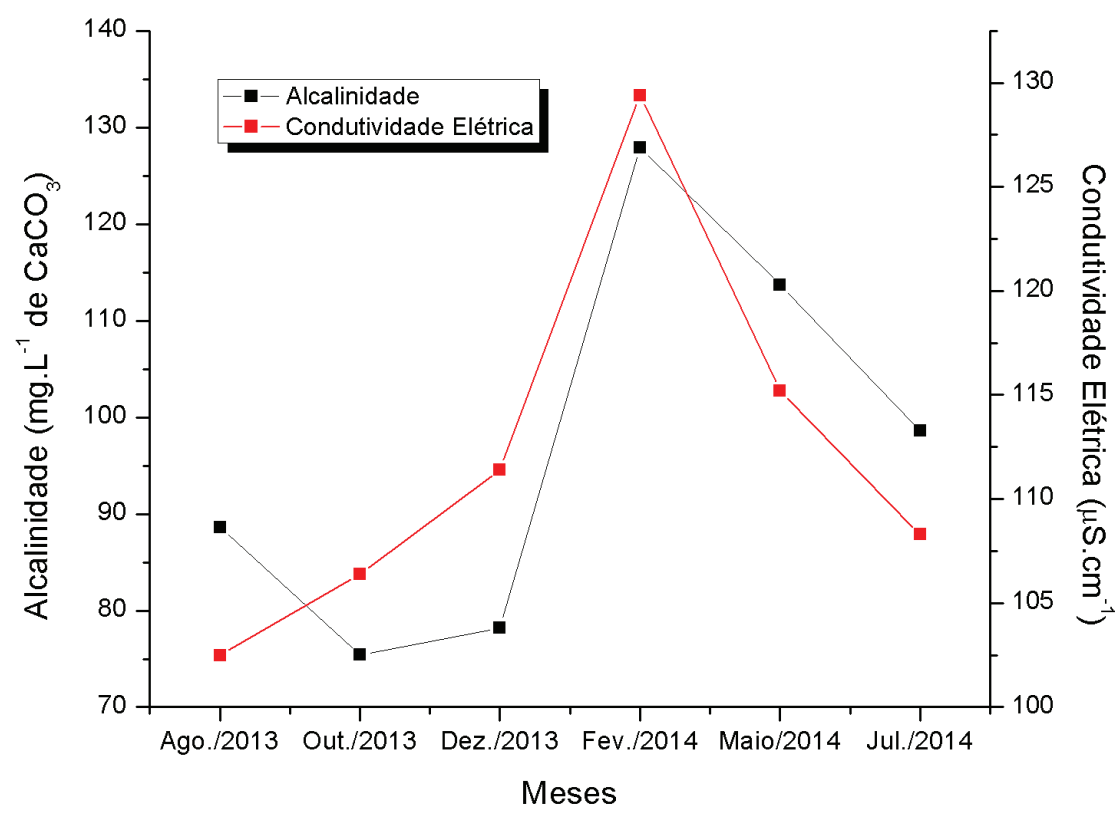

Figura 4. Correlação entre os valores obtidos para a alcalinidade e condutividade elétrica

Os resultados para a amônia não ionizada $\left(\mathrm{NH}_{3}\right)$ variaram entre 0,05 a 1,77 mg.L.-1 , sendo registrado o menor valor em out./2013 na saída do viveiro 3, e o maior valor também foi registrado em out./2013, na saída do viveiro 1 , havendo diferença significativa entre as médias (Tabela 2). Os valores obtidos para a amônia não ionizada estão dentro do padrão exigido pela resolução Conama 357/2005 que é de 2,18 mg.L $\mathrm{L}^{-1}$ para corpos d'água utilizados para pesca ou criação intensiva de organismos aquáticos para fins de consumo.

A determinação dos níveis de $\mathrm{NH}_{3}$ na água é necessária, pois o seu aumento ocasiona a redução de sua excreção pelo peixe, e o consequente acúmulo desse metabólito em seus tecidos (BALDISSEROTO, 2013b). Muitos estudos confirmam que a $\mathrm{NH}_{3}$ é a forma mais tóxica da amônia total $\left(\mathrm{NH}_{4}^{1+}+\mathrm{NH}_{3}\right)$ presente na água. Essa toxicidade é pela sua baixa capacidade de difusão por meio das membranas celulares, o que causa danos ao epitélio branquial e, consequentemente, 
compromete os mecanismos osmorreguladores (DUTRA et al., 2016). Ainda de acordo com Kubitza (2003), valores de $\mathrm{NH}_{3}$ acima de 0,20 mg.L $\mathrm{L}^{-1}$ são suficientes para induzir toxicidade crônica e levar à diminuição do crescimento e tolerância dos peixes a doenças; entre 0,70 e $2,40 \mathrm{mg} . \mathrm{L}^{-1}$ podem ser letais e quando expostos frequentemente a concentrações de $\mathrm{NH}_{3}$ acima de $0,02 \mathrm{mg}$. $\mathrm{L}^{-1}$ pode haver intensa irritação e inflamação nas brânquias.

A amônia ionizada $\left(\mathrm{NH}_{4}{ }^{1+}\right)$ variou entre 0,05 a 1,88 mg. $\mathrm{L}^{-1}$, sendo ambos os valores registrados em out./2013, o valor mínimo na saída do viveiro 3, e o valor máximo na saída do viveiro 1 (Tabela 1), havendo diferença significativa apenas entre as médias do mês de dez./2013 e fev./2014 (Tabela 2).

$\mathrm{Na}$ piscicultura intensiva, a principal fonte de compostos nitrogenados incorporados à água é a alimentação, no início do cultivo, quando a biomassa é ainda pequena; observam-se baixos níveis de amônia total, compostos resultantes do catabolismo das proteínas, sendo que tais componentes aumentam proporcionalmente ao aumento da quantidade de alimento fornecido e da biomassa (HURVITZ; BERCOVIER; RIJN, 1997), que foi registrado nos resultados obtidos em fev./2014 (início do novo ciclo de produção), em que os valores de $\mathrm{NH}_{3} \mathrm{e} \mathrm{NH}_{4}{ }^{1+}$ foram baixos em relação ao mês de dez./2013 (fim do ciclo de produção), mês de valor mais elevado para a amônia ionizada e não ionizada (Tabela 1).

Os valores obtidos de nitrito $\left(\mathrm{NO}_{2}{ }^{1-}\right)$ variaram entre 0,0 a $0,35 \mathrm{mg} \cdot \mathrm{L}^{-1}$, e sua presença não foi detectada em out./2013 na saída do viveiro 3 e no mês de fev./2014 nos pontos amostrados do viveiro 5, e o valor máximo $\left(0,35 \mathrm{mg} . \mathrm{L}^{-1}\right)$ foi registrado em ago./2013 na represa de abastecimento (Tabela 1). Segundo a resolução Conama $357 / 2005$ os valores de $\mathrm{NO}_{3}{ }^{1 \cdot}$ não devem exceder a $1 \mathrm{mg} . \mathrm{L}^{-1}$, portanto os valores registrados neste estudo estão dentro dos padrões estipulados para aquicultura.

$\mathrm{O} \mathrm{NO}_{2}{ }^{1-}$ é proveniente da incompleta oxidação da amônia total a nitrato (ATWOOD; FONTENOT; TOMASSO 2001), os quais em sistemas de cultivo de peixes sem recirculação, disposição sequencial ou baixa renovação de água são provenientes do catabolismo de compostos nitrogenados, e também pela decomposição de alimentos não digeridos e outros resíduos orgânicos. Para os peixes, $\mathrm{o} \mathrm{NO}_{2}{ }^{1-}$ é muito tóxico, pois se combina à hemoglobina no sangue, originando a meta-hemoglobina, que confere coloração amarronzada ao sangue, matando o peixe por asfixia (LIMA; 
HOLANDA; RIBEIRO, 2008).

$\mathrm{Na}$ Piscicultura Santa Helena, a relação entre o $\mathrm{OD}$ e o $\mathrm{NO}_{2}{ }^{1 \cdot}$ foi significativa durante todo o ciclo de cultivo (Figura 5), onde os teores máximos de $\mathrm{NO}_{2}{ }^{1 \cdot}$, e mínimo de OD ocorreram em dez./2013, porém o início dessa correlação ficou mais acentuado no início da fase de engorda (out./2013).

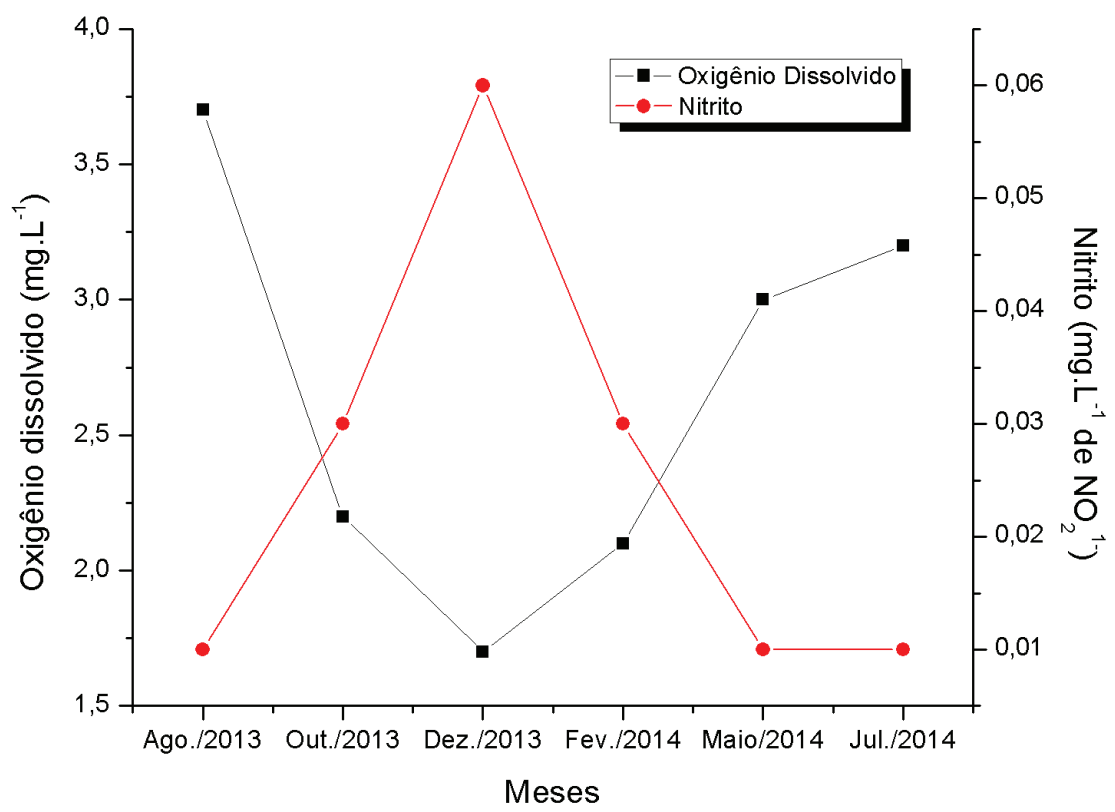

Figura 5. Variação do nitrito em função do oxigênio dissolvido

O fósforo total $\left(\mathrm{P}_{\mathrm{t}}\right)$, variou entre 0,0 a $2,0 \mathrm{mg} \cdot \mathrm{L}^{-1}$, não sendo detectado em fev./2014 na entrada do viveiro 1 e nos pontos amostrados no viveiro 5, o que pode estar relacionado com a despesca e posterior calagem realizada no mês de jan./2014. O valor máximo (2,0 mg..-1 $)$ foi verificado em dez./2013 na entrada do viveiro 3, o que pode estar relacionado com a finalização do ciclo de produção do tambaqui, pois, este valor está associado ao arraçoamento no ciclo final do cultivo, de modo que o viveiro 3 foi o que obteve os maiores índices médios de fósforo (Tabelas 1 e 2). 
Apesar de em alguns pontos de coleta os valores de $\mathrm{P}_{\mathrm{t}}$ estarem dentro dos valores exigidos pela resolução Conama 357/2005, estes em sua maioria estão fora dos valores estabelecidos pela referida legislação que determina concentração máxima de $0,05 \mathrm{mg} \cdot \mathrm{L}^{-1}$.

O $\mathrm{P}_{\mathrm{t}}$ também constitui um dos principais nutrientes para os processos biológicos; é um dos denominados macronutrientes, por ser exigido também em grandes quantidades pelas células (animais e vegetais) e, por ser nutriente para processos biológicos. O excesso de fósforo, por outro lado, conduz a processos de eutrofização do meio aquático, que tem como consequência o crescimento desordenado e bloom de algas (PEREIRA; RIBEIRO FILHO, 2004), o que foi observado em outubro/2013 subentendendo-se que os valores de $\mathrm{P}_{\mathrm{t}}$ provavelmente eram mais elevados, no entanto foram absorvidos pelas florações.

De acordo com Salati (2001) e Nobre, Sampaio e Salazar (2007), o cenário de precipitações pluviométricas na Região Amazônica apresenta uma sazonalidade que concentra alto índice pluviométrico em determinados meses do ano. Estudos realizados por Franca (2015) apontaram que o período mais chuvoso para o Estado de Rondônia são os meses de dezembro, janeiro e fevereiro e os meses de seca são junho, julho e agosto; tais informações corroboram os resultados obtidos nesta pesquisa (Figura 6). Franca (2015) também destaca que a variabilidade interanual da pluviosidade em Rondônia indica alternância natural entre anos habituais (normais), secos e chuvosos, com destaque para os extremos de chuva, entretanto, vem ocorrendo secas severas ou inundações das bacias hidrográficas da região. Segundo Salati (2001), tais alterações no equilíbrio dinâmico da atmosfera amazônica estão relacionadas a variações climáticas de causas naturais, tais como os fenômenos $\mathrm{El}$ Niño e La Niña. 


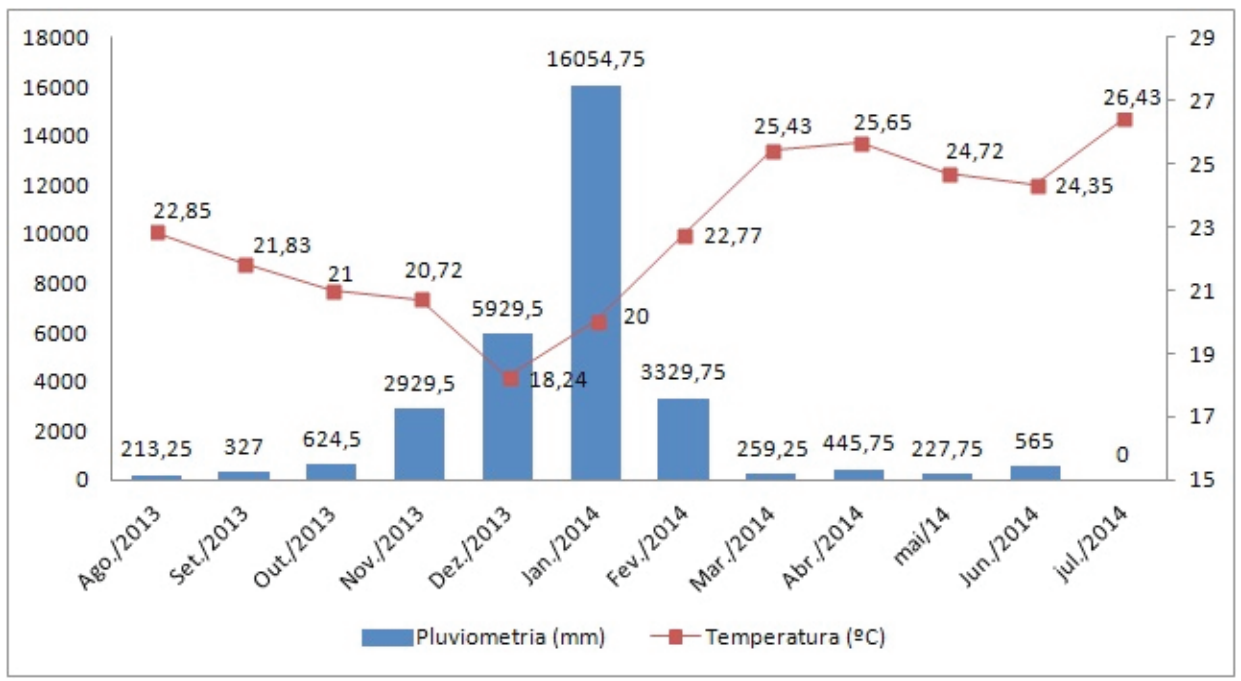

Figura 6. Dados pluviométricos e da temperatura na região do município de Alvorada d' Oeste Fonte: Dados coletados em jan./2015 na estação meteorológica A939 INMET.

\section{CONSIDERAÇÕES FINAIS}

O manejo nos viveiros da Piscicultura Santa Helena, bem como o sistema de abastecimento interligado (tipo cascata) e a sazonalidade da pluviosidade na região influenciaram direta e indiretamente na variação dos parâmetros limnológicos, sendo de suma importância para o piscicultor não só conhecer, como também saber lidar com as mudanças e a dinâmica de tais parâmetros para o melhor desenvolvimento da atividade piscícola, e também se recomenda não só para a Piscicultura Santa Helena como para outros empreendimentos aquícolas em geral uma estrutura de abastecimento e drenagem de viveiros de forma individualizada para melhor controle da produção, assim como a necessidade de adoção sistemática de boas práticas de manejo a fim de melhorar a qualidade ambiental

\section{AGRADECIMENTOS}

Ao CNPq, a Universidade Federal de Rondônia, a Nutrizon Alimentos Ltda. e ao senhor Lázaro de Jesus Ramos, proprietário da Piscicultura Santa Helena. 


\section{REFERÊNCIAS}

ARIDE, P. H. R.; ROUBACH, R.; VAL, A. L. Tolerance response of tambaqui Colossoma macropomum (Cuvier) to water pH. Aquaculture Research, v. 38, n. 6, p. 588-594, 2007.

ATWOOD, H. L.; FONTENOT, Q. C.; TOMASSO, J. R. Toxicity of nitrite to Nile tilapia: effect of fish size and environmental chloride. North American Journal of Aquaculture, v. 63, n. 1, p. 49-51, 2001.

BALDISSEROTTO, B. Resíduos nitrogenados. In: BALDISSEROTTO, B. Fisiologia de peixes aplicada à piscicultura. 3. ed. Santa Maria: Ed. da UFSM. 2013. cap. 6, p. 139-156a.

BALDISSEROTTO, B. Crescimento. In: BALDISSEROTTO, B. Fisiologia de peixes aplicada à piscicultura. 3. ed. Santa Maria: Ed. da UFSM. 2013. cap. 9, p. 231-342b.

BARBOSA, E. B.; SOUSA NETO, R. F. Piscicultura no distrito agropecuário da Suframa: produção do peixe tambaqui no município de Rio Preto da Eva - AM. Observatorio de la Economía Latinoamericana, n. 194, 2014. Disponível em: < http://www. eumed.net/cursecon/ecolat/br/14/piscicultura-suframa.html>. Acesso em 18 jan. 2017.

BOYD, C. E. Bottom soil and water quality management in shrimp ponds. Journal of Applied Aquaculture, v. 13, n. 1-2, p. 11-33, 2003.

BOYD, C. E; TUCKER, C. S. Pond aquaculture water quality managment. Boston: Kluwer Academic Pulishers, 1992, 700p.

CECCARELLI, P. S.; SENHORINI, J. A.; VOLPATO, G. Dicas em Piscicultura: perguntas e respostas. Santana, Botucatu, SP. 2000. p. 247.

CETESB - Companhia Ambiental do Estado de São Paulo. Guia nacional de coleta e preservação de amostras: água, sedimento, comunidades aquáticas e efluentes líquidos. São Paulo: CETESB, 2011. 326 p. 
CONAMA - Conselho Nacional do Meio Ambiente. Ministério do Meio Ambiente; Resolução No 357/05 - Dispõe sobre a classificação dos corpos de água e diretrizes ambientais para o seu enquadramento, bem como estabelece as condições e padrões de lançamento de efluentes, e dá outras providências. Brasília (Brasil): Ministério do Meio Ambiente; 2005. Disponível em: < http://www.mma.gov.br/port/conama/ legiabre.cfm?codlegi=459> . Acesso em: $10 \mathrm{dez} .2016$.

CRAEF, E. W.; RESENDE, E. K. D.; PETRY, P.; STORTI FILHO, A. Policultivo de matrinchã (Brycon sp.) e jaraqui (Semaprochilodus sp.) em pequenas represas. Acta Amazônica, v. 17, p. 33-42, 1987.

DUTRA, F. M.; FORNECK, S. C.; BRAZÃO, C. C.; FREIRE, C. A.; BALLESTER, E. L. C. Acute toxicity of ammonia to various life stages of the Amazon river prawn, Macrobrachium amazonicum, Heller, 1862. Aquaculture, n. 453, p. 104-109, 2016.

ELER, M. N.; MILLANI, T. J. Métodos de estudos de sustentabilidade aplicados à aquicultura. Revista Brasileira de Zootecnia. v. 33, p. 33-44, 2007

FAO - Organización de las Naciones Unidas para la Alimentación y la Agricultura. El estado mundial de la pesca y la acuicultura: oportunidades y desafíos. Roma. 2014. Disponível em < http://www.fao.org/3/a-i3720s.pdf> Acesso em 05 nov. 2016.

FARIA, R. H. S.; MORAES, M.; SORANNA, M. R. G. S.; SALLUM, W. B. Manual de criação de peixes em viveiro. Brasília: Codevasf. 2013: 136 p.

FERNANDES, L. C.; GUIMARÃES, S. C. Governo do Estado de Rondônia. Secretaria de Estado do Desenvolvimento Ambiental. Atlas Geoambiental de Rondônia. Edição digital. SEDAM, Porto Velho, CD ROM, 2003.

FRANCA, R. R. Climatologia das chuvas em Rondônia - período 1981-2011, Geográficas, v. 11, n. 1, p. 44-58, 2015.

HURVITZ, A.; BERCOVIER, H.; RIJN, J. Van. Effect of ammonia on the survival and the immune response of rainbow trout (Oncorbynchus mykiss, Walbaum) vaccinated against Streptococcus iniae. Fish \& Shellfish Imunology, v.7, p.45-53, 1997. 
IBGE. Produção da Pecuária Municipal. Brasil: IBGE, 2013. Disponível em: < http:// ftp.ibge.gov.br/Producao_Pecuaria/Producao_da_Pecuaria_Municipal/2013/ ppm2013.pdf>. Acesso 25 out. 2016.

IBGE. Produção da Pecuária Municipal. Brasil: IBGE, 2014. Disponível em: < http:// ftp.ibge.gov.br/Producao_Pecuaria/Producao_da_Pecuaria_Municipal/2014/ ppm2014.pdf $>$. Acesso 25 out. 2016.

ITUASSU,D. R.; SANTOS, G. R. S.; ROUBACH, R.; PEREIRA-FILHO, M. Desenvolvimento de tambaqui submetido a períodos de privação alimentar. Pesquisa Agropecuária Brasileira, v. 39, p. 1199-1203, 2004.

KUBITZA, F. Qualidade da água na produção de peixes. Panorama da Aquicultura, Jundiaí-SP, v. 8, n. 47, p. 34-43, 2003.

LIMA, C. L.; HOLANDA, E. D.; RIBEIRO, L. P. Doença no sangue marrom de tilápias, Oreochromis $s p$, produzidas em recirculação. Revista Brasileira de Higiene e Sanidade Animal. v. 2, n. 1, p. 35 - 42, 2008.

MINUCCI, L. V.; PINESE, J. F.; ESPÍNDOLA, E. L. G. Análise limnológica de sistema semi-intensivo de criação de Leporinus macrocephalus (Pisces, Anostomidade). Bioscience Journal, v. 21, n. 1, p. 123-131, 2005.

NOBRE, C. A.; SAMPAIO, G.; SALAZAR, L. Mudanças climáticas e Amazônia. Ciência e Cultura, v. 59, n. 3, p. 22-27, 2007.

OLIVEIRA, R. P. C.; SILVA, P. C.; BRITO, P. P.; GOMES, J. P.; SILVA, R. F.; SILVEIRA FILHO, P. R.; ROQUE, R. S. Variáveis hidrológicas físico-químicas na criação da tilápiado-nilo no sistema raceway com diferentes renovações de água. Ciência Animal Brasileira, v. 11, p. 482-487, 2010.

PEREIRA, D. A. S.; QUEROL, M. V. M.; GRALHA, T. S.; MACHADO, M. M.; PESSANO, E. F. C.; DE OLIVEIRA, L. F. S. Programa Proext/Mec (Fase 3) de Capacitação de Pescadores e Produtores Rurais Água na Qualidade do Cultivo (1). Anais do Salão Internacional de Ensino, Pesquisa e Extensão, v. 7, n. 3, 2016. 
PEREIRA, J. M. A.; RIBEIRO FILHO, R. A. Efeitos da excreção de tilápias (Tilápia rendalli e Oreocbromis niloticus) em reservatórios e viveiros de Piscicultura. In: ESPÍNDOLA, E. L. G.; SCHALCH, V. (Org.) Bacias Hidrográficas: diversas abordagens em pesquisa, São Carlos: RIMA. 2004: 99-110.

QUEIROZ, J. F.; BOEIRA, R. C. Calagem e Controle da Acidez dos Viveiros de Aquicultura. Jaguariúna: Embrapa Meio Ambiente. 2006: 8 p.

RESENDE, E. K. D.; GRAEF, E. W.; ZANIBONI FILHO, E.; PAIXÃO, A. M.; STORTI FILHO, A. Avaliação do crescimento e produção de jaraquis (Semaprochilodus $s p p$.), em açude de igarapé de terra firme nos arredores de Manaus, Amazonas. Acta Amazônica, v. 15, n. 1-2, p. 19-34, 1985.

SALATI, E. Mudanças climáticas e o ciclo hidrológico naAmazônia. In: FLEISCHRESSER, V. (ed.). Causas e Dinâmica do Desmatamento na Amazônia. Ministério do Meio Ambiente, Brasília, DF, p.153-172, 2001.

SILVA, V. K.; FERREIRA, M. W.; LOGATO, P. V. R. Qualidade da água na Piscicultura. Boletim de Extensão da UFLA, Lavras, MG, n. 94, 2001.

TUNDISI, J. G.; STRASKRABA, M. Strategies for building partnerships in the context of river basin management: the role of ecotechnology and ecological engineering, in lakes \& reservoirs. Research and Management. v. 1, p. 31-38, 1995.

WATANABE, A. et al. Princípios técnicos de piscicultura. Dossiê Técnico, USP/DT, São Paulo-SP. 2007: 19 p.

Recebido em: 2016-04-23

Aceito em: 2017-02-10 